\title{
ENTIRE FUNCTIONS THAT SHARE A POLYNOMIAL WITH ONE OF THEIR DERIVATIVES*
}

\author{
JIAN-PING WANG
}

\begin{abstract}
In this paper, we investigate the entire functions that share a polynomial with one of their derivatives and prove several theorems which generalize the main results given by L. Z. Yang in [14].
\end{abstract}

\section{Introduction and results}

In this paper, the term "meromorphic" will mean meromorphic in the whole complex plane. Let $f$ and $g$ be two nonconstant meromorphic functions, and let $P$ be a polynomial or a finite value. We say that $f$ and $g$ share $P$ IM (ignoring multiplicities) provided that $f-P$ and $g-P$ have the same zeros. If $f-P$ and $g-P$ have the same zeros with the same multiplicities, then we say that $f$ and $g$ share $P \mathrm{CM}$ (counting multiplicities). It is assumed that the reader is familiar with the usual notations and fundamental results of R. Nevanlinna's theory of meromorphic functions, as found in [6] and [13].

Rubel and Yang seems to have been the first to study the entire functions that share values with their derivatives. They proved

THEOREM A (see [11]). If a nonconstant entire function $f$ share two distinct, finite values $C M$ with $f^{\prime}$, then $f \equiv f^{\prime}$.

Since then, shared value problems on entire functions, especially, the case when $f$ and its derivatives share values have attracted considerable attention during the last quarter of century or so, and a number of results have been obtained, some of the main results being due to G. Gundersen, E. Mues and C. C. Yang (see [1-2], [7-8], [10], [12], [14-16] etc).

In 2000, Li and Yang replaced $f^{\prime}$ with $f^{(k)}(k \geq 1)$ in Theorem $\mathrm{A}$, and proved the following results.

2000 Mathematics Subject Classification: 30D35, 30D20.

Keywords: meromorphic function, entire function, shared value.

* Project supported by the NSFC (Grant No. 10371065) and the Zhejiang Provincial NSF (Grant No. M103006) of China.

Received October 10, 2003; revised March 3, 2004. 
THEOREM B (see [8]). Let $f$ be a nonconstant entire function, and let $k$ be a positive integer. If $f$ and $f^{(k)}$ share two distinct, finite values $I M$, then $f \equiv f^{(k)}$.

Obviously, the conclusion of Theorem B is, in general, not true if $f$ and $f^{(k)}$ share only one value CM. It is natural to ask, however, what can be said when a nonconstant entire function $f$ shares one finite value CM with one of its derivatives $f^{(k)}(k \geq 1)$ ? This problem was studied by authors such as Jank, Mues and Volkmann, R. Brück, L. Z. Yang, Q. C. Zhang etc., and some results have been obtained under various extra conditions, respectively (see [1-2], [7, 8], [12], [14-16]).

In particular, we shall refer to the following results on this topic.

THEOREM C (see [1]). Let $f$ be a nonconstant entire function. If $f$ and $f^{\prime}$ share the value $1 C M$, and if $N\left(r, 0, f^{\prime}\right)=S(r, f)$, then

$$
\frac{f^{\prime}-1}{f-1}=c,
$$

where $c$ is a non-zero constant.

THEOREM D (see [14]). Let $f$ be a nonconstant entire function of finite order, and let $a \neq 0$ be a finite constant. If $f$ and $f^{(k)}$ share the value a $C M$, then

$$
\frac{f^{(k)}-a}{f-a}=c
$$

where $c$ is some non-zero constant and $k$ a positive integer.

In this paper, we prove that Theorem $\mathrm{D}$ still holds if the constant $a$ is replaced by a polynomial $Q(z)$, that is

THEOREM 1. Let $f$ be a nonconstant entire function of finite order, let $Q(z)$ be a polynomial with degree $q \geq 1$, and let $k$ be a positive integer. If $f$ and $f^{(k)}$ share $Q(z) C M$, then for some non-zero constant $c$, we have

$$
\frac{f^{(k)}-Q}{f-Q}=c
$$

As usual, we say that a finite value $z_{0}$ is a fixed point of a function $f$ if $f\left(z_{0}\right)=z_{0}$. So from Theorem 1 , we can easily obtain the following

COROLlaRY. Let $f$ be a nonconstant entire function of finite order, and let $k$ be a positive integer. If $f$ and $f^{(k)}$ have the same fixed points with the same multiplicities, then

$$
\frac{f^{(k)}-z}{f-z}=c
$$

for some non-zero constant $c$. 


\section{Lemmas}

Lemma 1 (see [3]). Let $F$ be a nonconstant meromorphic function of finite order $\rho$, let $\varepsilon>0$ be a given constant, and let $k$ be a positive integer. Then there exists a set $E \subset[0,2 \pi)$ that has linear measure zero, such that if $\psi_{0} \in[0,2 \pi)-E$, then there is a constant $R_{0}=R_{0}\left(\psi_{0}\right)>0$ such that for all $z$ satisfying $\arg z=\psi_{0}$ and $|z| \geq R_{0}$, we have $\left|F^{(k)}(z) / F(z)\right| \leq|z|^{k(\rho-1+\varepsilon)}$.

Lemma 2 (see [14, Lemma 2]). Let $F$ be an entire function, and let $k$ be a positive integer. Suppose that $\left|F^{(k)}(z)\right|$ is unbounded on some ray $\arg z=\phi$. Then there exists an infinite sequence of points $z_{n}=r_{n} e^{i \phi}$ where $r_{n} \rightarrow+\infty$, such that $F^{(k)}\left(z_{n}\right) \rightarrow \infty$ and

$$
\left|\frac{F\left(z_{n}\right)}{F^{(k)}\left(z_{n}\right)}\right| \leq(1+o(1))\left|z_{n}\right|^{k}
$$

as $z_{n} \rightarrow \infty$.

Lemma 3 (see [5, p. 119]). Suppose that $f(z)$ is an entire function such that $f(z) / z^{n}$ is bounded for $|z| \geq R$, where $R$ is a positive number. Then $f(z)$ is a polynomial of degree at most $n$.

Now we state the main lemma of this paper which has independent interest and, also is an improvement of [14, Theorem 1] and [2, Lemma 1].

Lemma 4. Let $P(z), Q(z)$ be two nonconstant polynomials, and let $q$ be the degree of $Q(z)$. Then for any positive integer $k$, every entire solution $F$ of the differential equation

$$
F^{(k)}-F e^{P}=Q-Q^{(k)}
$$

has infinite order.

Proof. We shall prove Lemma 4 by contradiction. Assume that Lemma 4 is not true, namely, suppose that $F$ is an entire solution of the Eq. (2.1) that has finite order $\rho$. Let $\varepsilon>0$ be any given constant. Then from Lemma 1, there exists a set $E \subset[0,2 \pi)$ that has linear measure zero, such that if $\psi_{0} \in[0,2 \pi)-E$, then there is a constant $R_{0}=R_{0}\left(\psi_{0}\right)>0$ such that for all $z$ satisfying $\arg z=\psi_{0}$ and $|z| \geq R_{0}$, we have

$$
\left|\frac{F^{(k)}(z)}{F(z)}\right| \leq|z|^{k(\rho-1+\varepsilon)} .
$$

Now suppose that $\theta$ is any real number that satisfies $\theta \in[0,2 \pi)-E$, and for every $\alpha>0$,

$$
\frac{\left|e^{P\left(r e^{i \theta}\right)}\right|}{r^{\alpha+q}} \rightarrow+\infty
$$


as $r \rightarrow+\infty$. Writing (2.1) as

$$
\frac{F^{(k)}}{\left(Q-Q^{(k)}\right) F}-\frac{e^{P}}{Q-Q^{(k)}}=\frac{1}{F} \text {. }
$$

Since (2.2) holds, thus for all $z=r e^{i \theta}$ with sufficiently large real number $r$, and for any positive number $\alpha>\max \{0, k(\rho-1)\}$, we have

$$
\left|\frac{F^{(k)}(z)}{F(z)}\right| \frac{1}{|z|^{\alpha+q}} \leq|z|^{k(\rho-1+\varepsilon)-\alpha-q} .
$$

Since $Q$ is a polynomial with degree $q(\geq 1)$, which means that $Q^{(q)}(0) \neq 0$, so we can write

$$
Q(z)=\frac{Q^{(q)}(0)}{q !} z^{q}+\frac{Q^{(q-1)}(0)}{(q-1) !} z^{q-1}+\cdots+Q^{\prime}(0) z+Q(0) .
$$

By (2.3), (2.4), (2.5) and (2.6), we obtain

$$
\begin{aligned}
\frac{1}{r^{\alpha}}\left|\frac{1}{F\left(r e^{i \theta}\right)}\right| & =\frac{1}{r^{\alpha}} \frac{1}{\left|Q\left(r e^{i \theta}\right)-Q^{(k)}\left(r e^{i \theta}\right)\right|}\left|\frac{F^{(k)}\left(r e^{i \theta}\right)}{F\left(r e^{i \theta}\right)}-e^{P\left(r e^{i \theta}\right)}\right| \\
& \geq \frac{q !}{2\left|Q^{(q)}(0)\right|} \frac{1}{r^{\alpha+q}}\left(\left|e^{P\left(r e^{i \theta}\right)}\right|-\left|\frac{F^{(k)}\left(r e^{i \theta}\right)}{F\left(r e^{i \theta}\right)}\right|\right) \rightarrow+\infty
\end{aligned}
$$

as $r \rightarrow+\infty$. From which we can deduce that

$$
F\left(r e^{i \theta}\right) \rightarrow 0 \quad \text { as } r \rightarrow+\infty .
$$

Next we suppose that $\phi$ is any real number that satisfies $\phi \in[0,2 \pi)$, and for every $\beta>0$,

$$
r^{\beta+q+k} e^{P\left(r e^{i \phi}\right)} \rightarrow 0
$$

as $r \rightarrow+\infty$.

Set $\quad R(z)=\sum_{j=0}^{q}\left(Q^{(q-j)}(0) /(q+k-j) !\right) z^{q+k-j} \quad$ and $\quad G(z)=F(z)-R(z)+$ $Q(z)$, then by $(2.6)$ we have $R^{(k)}(z)=Q(z)$. Now we assert that $\left|G^{(k)}(z)\right|=$ $\left|F^{(k)}(z)-Q(z)+Q^{(k)}(z)\right|$ is bounded on the ray $\arg z=\phi$. Assume the contrary, that is, suppose that $\left|G^{(k)}(z)\right|$ is unbounded on the $\operatorname{ray} \arg z=\phi$. Then from Lemma 2, there exists an infinite sequence of points $z_{n}=r_{n} e^{i \phi}$ where $r_{n} \rightarrow+\infty$, such that $G^{(k)}\left(z_{n}\right)=F^{(k)}\left(z_{n}\right)-Q\left(z_{n}\right)+Q^{(k)}\left(z_{n}\right) \rightarrow \infty$ and

$$
\left|\frac{G\left(z_{n}\right)}{G^{(k)}\left(z_{n}\right)}\right| \leq(1+o(1))\left|z_{n}\right|^{k} \quad \text { as } r_{n} \rightarrow+\infty \text {. }
$$

From (2.1) we have

$$
F^{(k)}(z)-Q(z)+Q^{(k)}(z)=F(z) e^{P(z)} .
$$

Since $F^{(k)}\left(z_{n}\right)-Q\left(z_{n}\right)+Q^{(k)}\left(z_{n}\right)=G^{(k)}\left(z_{n}\right) \rightarrow \infty$, it follows from (2.8) and (2.10) that $F\left(z_{n}\right) \rightarrow \infty$. For the proof of the above assertion, we shall consider the following two cases. 
CASE 1. There exist a positive number $\alpha_{0}>2\left|Q^{(q)}(0)\right| /(q+k)$ ! and an infinite subsequence, say $z_{n_{j}}$, of $z_{n}$, such that $\left|F\left(z_{n_{j}}\right)\right| \geq \alpha_{0}\left|z_{n_{j}}\right|^{q+k}$ for $j=1,2, \ldots$. Then for any positive number $\beta$ and sufficiently large integer $j$, we have

$$
\left|F\left(z_{n_{j}}\right)\right| \geq \alpha_{0}\left|z_{n_{j}}\right|^{q+k} \geq \frac{\left\{2\left|Q^{(q)}(0)\right| /(q+k) !\right\}\left|z_{n_{j}}\right|^{q+k}}{1-\left(1 /\left|z_{n_{j}}\right|^{\beta}\right)}=\frac{2\left|Q^{(q)}(0)\right|}{(q+k) !} \frac{\left|z_{n_{j}}\right|^{q+\beta+k}}{\left|z_{n_{j}}\right|^{\beta}-1},
$$

That is

$$
\frac{1}{\left|F\left(z_{n_{j}}\right)\right|-\left\{2\left|Q^{(q)}(0)\right| /(q+k) !\right\}\left|z_{n_{j}}\right|^{q+k}} \leq \frac{\left|z_{n_{j}}\right|^{\beta}}{\left|F\left(z_{n_{j}}\right)\right|} .
$$

From (2.11), (2.9), (2.6), the fact $\left|F\left(z_{n_{j}}\right)\right| \geq \alpha_{0}\left|z_{n_{j}}\right|^{q+k}$ as well as the definitions of $G(z)$ and $R(z)$ we have

$$
\begin{aligned}
& (1+o(1))\left|z_{n_{j}}\right|^{-k} \\
& \leq\left|\frac{G^{(k)}\left(z_{n_{j}}\right)}{G\left(z_{n_{j}}\right)}\right|=\frac{\left|G^{(k)}\left(z_{n_{j}}\right)\right|}{\left|F\left(z_{n_{j}}\right)-R\left(z_{n_{j}}\right)+Q\left(z_{n_{j}}\right)\right|} \\
& \leq \frac{\left|G^{(k)}\left(z_{n_{j}}\right)\right|}{\left|F\left(z_{n_{j}}\right)\right|-\left|\left(Q^{(q)}(0) /(q+k) !\right) z_{n_{j}}^{q+k}+\cdots+\left(Q^{\prime}(0) /(k+1) !\right) z_{n_{j}}^{k+1}+(Q(0) / k !) z_{n_{j}}^{k}-Q\left(z_{n_{j}}\right)\right|} \\
& \leq \frac{\left|G^{(k)}\left(z_{n_{j}}\right)\right|}{\left|F\left(z_{n_{j}}\right)\right|-2\left(\left|Q^{(q)}(0)\right| /(q+k) !\right)\left|z_{n_{j}}\right|^{q+k}} \leq \frac{\left|G^{(k)}\left(z_{n_{j}}\right)\right|}{\left|F\left(z_{n_{j}}\right)\right|}\left|z_{n_{j}}\right|^{\beta},
\end{aligned}
$$

and thus we obtain

$$
(1+o(1))\left|z_{n_{j}}\right| \leq\left|\frac{G^{(k)}\left(z_{n_{j}}\right)}{F\left(z_{n_{j}}\right)}\right|\left|z_{n_{j}}\right|^{\beta+k+1} .
$$

Now we rewrite $(2.10)$ as

$$
\frac{G^{(k)}(z)}{F(z)}=e^{P(z)}
$$

By considering (2.12), and (2.13), we can deduce that

$$
r_{n_{j}}^{\beta+k+1} e^{P\left(r_{n_{j}} j^{i \phi}\right)} \rightarrow \infty \quad \text { as } r_{n_{j}} \rightarrow+\infty,
$$

which contradicts the assumption (2.8).

CASE 2. For every given positive number $\alpha>2\left|Q^{(q)}(0)\right| /(q+k)$ !, the inequality $\left|F\left(z_{n}\right)\right|<\alpha\left|z_{n}\right|^{q+k}$ holds except for at most finitely many positive integers $n$.

In this case, we choose $\alpha=2\left|Q^{(q)}(0)\right|$. Then for any positive number $\beta>q+k$ and positive integer $n$ that is large enough, we obtain 


$$
\left|z_{n}\right|^{\beta}\left|\frac{G^{(k)}\left(z_{n}\right)}{F\left(z_{n}\right)}\right| \geq \frac{\left|G^{(k)}\left(z_{n}\right)\right|}{2\left|Q^{(q)}(0)\right|}\left|z_{n}\right|^{\beta-q-k} \rightarrow+\infty \quad \text { as } r_{n} \rightarrow+\infty .
$$

It follows from (2.13) and (2.14) that $\left|z_{n}\right|^{\beta}\left|e^{P\left(z_{n}\right)}\right| \rightarrow+\infty$, which contradicts the assumption (2.8) again.

Now the assertion that $\left|G^{(k)}(z)\right|$ is bounded on the ray $\arg z=\phi$ has been confirmed. From this assertion and the formula $G^{(k-1)}(z)=G^{(k-1)}(0)+$ $\int_{0}^{z} G^{(k)}(\omega) d \omega$, we obtain that $\left|G^{(k-1)}(z)\right| \leq\left|G^{(k-1)}(0)\right|+M|z|$ for all $z$ satisfying $\arg z=\phi$, where $M=M(\phi)>0$ is some constant. Similarly, by integrating the function $G^{(k)} k$ times, we can deduce that for all $z$ satisfying $\arg z=\phi$,

$$
|G(z)| \leq|G(0)|+\left|G^{\prime}(0)\right||z|+\cdots+\left|G^{(k-1)}(0)\right||z|^{k-1}+M|z|^{k} .
$$

From the above and the definition of the function $R(z)$, it follows that for all $z$ satisfying $\arg z=\phi$,

$$
\begin{aligned}
|F(z)| & \leq|G(z)|+|R(z)|+|Q(z)| \\
& \leq|G(0)|+\left|G^{\prime}(0)\right||z|+\cdots+\left|G^{(k-1)}(0)\right||z|^{k-1}+M|z|^{k}+2 \frac{\left|Q^{(q)}(0)\right|}{(q+k) !}|z|^{q+k} \\
& =\left(2 \frac{\left|Q^{(q)}(0)\right|}{(q+k) !}+o(1)\right)|z|^{q+k}
\end{aligned}
$$

as $|z| \rightarrow+\infty$.

We now have shown that $(2.15)$ holds for any $\phi \in[0,2 \pi)$ with property (2.8), and that (2.7) holds for any $\theta \in[0,2 \pi)-E$ with property (2.3). Suppose that $\rho_{1}$ is a real number not less than $\rho$ which is the order of the function $F$. Then we can find a positive number $\alpha_{1}$ such that $\alpha_{1}<1 / \rho_{1}$. Noting that $F(z) / z^{q+k}$ is an entire function of finite order $\rho$ in $|z| \geq 1$, we can deduce that for every given constant $\varepsilon>0$, there exists a real number $r_{1}>1$ such that $\left|F(z) / z^{q+k}\right|<$ $\exp \left(\varepsilon r^{1 / \alpha_{1}}\right)$ as $|z|=r \geq r_{1}$.

Since $P(z)$ is a nonconstant polynomial, so there exist only finitely many real numbers in $[0,2 \pi)$ that do not satisfy either (2.3) or (2.8). Also we note that the set $E$ has linear measure zero, thus there exist a finite collection of real numbers $\theta_{j} \in[0,2 \pi)-E$ that satisfy either (2.3) or (2.8), where $\theta_{1}<\theta_{2}<\cdots<\theta_{n}=$ $\theta_{1}+2 \pi$, and $\alpha_{1} \pi / 2<\theta_{j+1}-\theta_{j} \leq \alpha_{1} \pi$ for $j=1, \ldots, n-1$. By the rays $\arg z=$ $\theta_{j}(j=1, \ldots, n)$, we divide the set $\left\{z \in C:|z| \geq r_{1}\right\}$ into sector domains $S_{j}=\{z \in$ $\left.C: \theta_{j} \leq \arg z \leq \theta_{j+1},|z| \geq r_{1}\right\} \quad(j=1, \ldots, n-1)$. In view of (2.7), (2.15) and the fact that $\left|F(z) / z^{q+k}\right|<\exp \left(\varepsilon r^{1 / \alpha_{1}}\right) \leq \exp \left(\varepsilon r^{\pi /\left(\theta_{j+1}-\theta_{j}\right)}\right)$ for $j=1, \ldots, n-1$ and $|z|=$ $r \geq r_{1}$, hence we can apply the Phragmén-Lindelöf theorem [9, Theorem 9.12] to the function $F(z) / z^{q+k}$ in every sector domain $S_{j}(j=1, \ldots, n-1)$, and deduce that there exist two positive constants $M_{0}$ and $r_{0}$ such that $|F(z)| \leq M_{0}|z|^{q+k}$ as $|z|=r \geq r_{0}$. From this and Lemma 3, we can conclude that $F(z)$ must be a polynomial of order at most $q+k$. However, this is impossible because $P(z)$ is a nonconstant polynomial in (2.1). This contradiction proves Lemma 4. 


\section{Proof of Theorem 1}

Since $f$ and $f^{(k)}$ share the polynomial $Q \mathrm{CM}$, and noting that $f$ is of finite order, so from the Hadamard factorization theorem we have

where $P(z)$ is a polynomial.

$$
\frac{f^{(k)}-Q}{f-Q}=e^{P(z)}
$$

Put $f-Q=F$, then we can see from (3.1) that $F$ is an entire solution of the differential equation

$$
F^{(k)}-F e^{P}=Q-Q^{(k)} .
$$

Noting that $F$ has finite order, and thus we can deduce from (3.2) and Lemma 4 that $P$ must be a constant. This proves Theorem 1 .

Acknowledgement. The author thank Professor Hong-xun Yi for his valuable advice about this paper. The author is also very grateful to the referee for his/her valuable comments and suggestions.

\section{REFERENCES}

[1] R. BRÜCK, On entire functions which share one value CM with their first derivative, Results in Math., 30 (1996), 21-24.

[2] G. G. Gundersen AND L. Z. YANG, Entire functions that share one value with one or two of their derivatives, J. Math. Anal. Appl., 233 (1998), 88-95.

[ 3 ] G. G. Gundersen, Estimates for the logarithemic derivatives of a meromorphic function, plus similar estimates, J. London Math. Soc., 37 (1988), 88-104.

[ 4 ] G. G. Gundersen, Finite order solution of second order linear differential equation, Trans. Amer. Math. Soc., 305 (1988), 415-429.

[ 5 ] T. W. Gamelin, Complex Analysis, in: Undergraduate Texts in Math., Springer-Verlag, New York, Inc., 2001.

[6] W. K. Hayman, Meromorphic Functions, Clarendon, Oxford, 1964.

[7] G. Jank, E. Mues and L. Volkmann, Meromorphe funktionen, die mit iher ersten und zweiten Ableitung einen endlichen Wert teilen, Complex Variables Theory Appl., 6 (1986), 51-71.

[ 8 ] P. Li AND C. C. YANG, When an entire function and its linear differential polynomial share two values, Illinois J. of Math., 44 (2000), 349-362.

[9] A. Markushevich, Theory of functions of a complex variable, Vol. 2, translated by R. Silverman, Prenctice-Hall, Englewood Cliffs, NJ. 1965.

[10] E. Mues And N. Steinmetz, Meromorphe funktionen, die mit ihrer ableitung werte teilen, Manuscripta Math., 29 (1979), 195-206.

[11] L. A. Rubel AND C. C. YANG, Values shared by an entire function an its derivative, in: J. D. Buckholtz, T. J. Suffridge (Eds.), Conference of Complex Analysis, University of Kentucky, 1976, in: Lecture Notes in Math., Vol. 599, Springer-Verlag, Berlin, 1977, pp. 101-103.

[12] Jian-Ping Wang AND Hong-Xun YI, Entire functions that share one value CM with their derivatives, J. Math. Anal. Appl., 277 (2003), 155-163.

[13] H. X. Yi And C. C. YAng, Uniqueness Theory of Meromorphic Functions, in: Pure Appl. Math. Monogr., Vol. 32, Science Press, Beijing, 1995. 
[14] L. Z. YANG, Solution of a differential equation and its applications, Kodai Math. J., 22 (1999), 458-464.

[15] H. L. Zhong, Entire functions that share one value with their derivatives, Kodai Math. J., 18 (1995), 250-259.

[16] Q. C. Zhang, The uniqueness of meromorphic functions with their derivatives, Kodai Math. J., 21 (1998), 179-184.

Department of Mathematics

Shaoxing College of ARts and Sciences

ShaOXING, ZheJIANG 312000

People's RePublic of China

e-mail: jpwang604@eyou.com 